\title{
Estresse ocupacional em equipes saúde da família certificadas e não certificadas com selo de qualidade assistencial
}

\author{
Occupational stress in family health teams certified and non-certified with assistance quality seal
}

Estrés laboral en equipos de salud familiar certificados y no certificados con sello de calidad asistencial

Luiza Ferreira Rigonatti Silva ${ }^{1}$ (1) Alessandro Rolim Scholze ${ }^{1}$ (D) Paloma de Souza Cavalcante Pissinati ${ }^{2}$ (1) Janaína Recanello Begui ${ }^{1}$ (D) Maynara Fernanda Carvalho Barreto ${ }^{1}$ (D) Maria José Quina Galdino ${ }^{1}$ [D

1. Universidade Estadual do Norte do Paraná. Bandeirantes, PR, Brasil.

2. Secretaria Municipal de Saúde de Rolândia. Rolândia, PR, Brasil.
Autor correspondente:

Maria José Quina Galdino.

E-mail: mjggaldino@gmail.com.

Recebido em 11/11/2020.

Aprovado em 17/03/2021.

\begin{abstract}
Resumo
Objetivo: comparar o estresse ocupacional em trabalhadores de equipes saúde da família certificadas e não certificadas com selo de qualidade assistencial pela Tutoria da Atenção Primária à Saúde. Métodos: estudo transversal realizado com 178 trabalhadores das equipes da Estratégia Saúde da Família de dois municípios do Paraná. Os dados foram coletados por um questionário de caracterização e a Job Stress Scale, e analisados descritiva e inferencialmente por meio de regressão logística bruta e ajustada. Resultados: os trabalhadores vinculados às equipes certificadas apresentaram chances significativamente maiores de alta demanda psicológica ( $\left.p<0,001 ; \mathrm{R}^{\mathrm{a}:} 4,164\right)$ e baixo apoio social $\left(p=0,048 ; O R^{\text {aj: }} 1,896\right)$ em relação aos das não certificadas. $O$ controle sobre $o$ trabalho não apresentou diferença significativa $\left(p=0,891 ; O R^{\text {al: }}: 1,047\right)$. Os participantes de equipes certificadas apresentaram maior chance de $j o b \operatorname{strain}\left(\mathrm{p}<0,001 ; O \mathrm{R}^{\mathrm{aj}}: 4,956\right)$ e entre aqueles de equipes não certificadas predominou o trabalho passivo ( $\left.p<0,001 ; O R^{\text {ai: }} 0,293\right)$. Conclusão: os trabalhadores de equipes de saúde com certificação de qualidade na prestação de serviços apresentaram maiores chances de estresse ocupacional em relação aqueles vinculados a equipes não certificadas. Implicações para a prática: torna-se premente que os modelos gerenciais de qualidade da assistência considerem a saúde dos trabalhadores envolvidos.
\end{abstract}

Palavras-chave: Estresse Ocupacional; Atenção Primária à Saúde; Pessoal de Saúde; Saúde do Trabalhador; Gestão da Qualidade.

\begin{abstract}
Objective: to compare occupational stress in workers of health team family certified and non-certified with quality care seal by the Tutoring Primary Health Care. Methods: cross-sectional study carried out with 178 workers from the Family Health Strategy teams from two municipalities in Paraná. The data were collected through a characterization questionnaire and the Job Stress Scale, and analyzed descriptively and inferentially, through unadjusted and adjusted logistic regression. Results: workers linked to certified teams had significantly higher chances of high psychological demand ( $<0.001 ; \mathrm{OR}: 3.781)$ and low social support $(p=0.030$; OR: 1.896) in relation to those from non-certified team. Control over work showed no significant difference $(p=0.891$ $O R^{\text {al }: ~ 1.047) . ~ P a r t i c i p a n t s ~ f r o m ~ c e r t i f i e d ~ t e a m ~ h a d ~ a ~ h i g h e r ~ c h a n c e ~ o f ~ j o b ~ s t r a i n ~}\left(p<0.001 ; O R^{\text {aj: }}: 4.956\right)$ and among those from noncertified team, passive work predominated ( $p<0.001 ; \mathrm{OR}^{\mathrm{a}}$ : 0.293$)$. Conclusion: workers of team with quality certification in the provision of services had a greater chance of occupational stress in relation to those linked to non-certified team. Implications for the practice: it is imperative that the management models of quality of care consider the health of the workers involved.
\end{abstract}

Keywords: Occupational Stress; Primary Health Care; Health Personnel; Occupational Health; Quality Management.

\section{REsumen}

Objetivo: comparar el estrés laboral en trabajadores de los equipos de salud familiar certificados y no certificados con sello de calidad en la atención de la Mentoría de Atención Primaria. Métodos: estudio transversal con 178 trabajadores de los equipos del plan Estrategia Salud de la Familia de dos municipios de Paraná. Los datos fueron recolectados a través de un cuestionario de caracterización y de la Job Stress Scale, por medio de análisis descriptivo e inferencial, mediante regresión logística bruta y ajustada. Resultados: los trabajadores vinculados a equipos certificados presentaron probabilidades significativamente mayores de alta demanda psicológica $(p<0,001 ;$ OR: 3,781$)$ y bajo apoyo social $(p=0,030 ;$ OR: 1,896$)$ en relación a los de equipos no certificados. El control sobre el trabajo no mostró diferencia significativa ( $p=0,891 ; \mathrm{OR}^{\mathrm{aj}}$ : 1,047$)$. Los participantes de equipos certificadas obtuvieron una mayor probabilidad de padecer tensión laboral $\left(p<0,001 ; O R^{\text {al: }} 4,956\right)$ y entre los de equipos no certificadas predominó el trabajo pasivo ( $\left.p<0,001 ; \mathrm{OR}^{\mathrm{a}}: 0,293\right)$. Conclusión: los trabajadores de equipos de salud con certificación de calidad en la prestación de servicios presentaban mayor probabilidad de estrés ocupacional en relación a los vinculados a equipos no certificados. Implicaciones para la práctica: es imperativo que los modelos de gestión de la calidad de la atención consideren la salud de los trabajadores involucrados.

Palabras-Clave: Estrés Laboral; Atención Primaria de Salud; Personal de Salud; Salud Laboral; Gestión de la Calidad. 


\section{INTRODUÇÃO}

A Atenção Primária à Saúde (APS) é uma estratégia consolidada e eficiente de organização dos sistemas de saúde, por preconizar o acesso universal aos serviços e a atenção integral com qualidade à população. ${ }^{1}$ A APS objetiva descentralizar os serviços, organizar o modelo assistencial, ser a provedora e coordenadora do cuidado, além de incorporar as diretrizes e princípios do Sistema Único de Saúde (SUS). ${ }^{2}$ Em busca do rompimento de um modelo hegemônico centrado na doença, em 1994, o Ministério da Saúde implantou a Estratégia Saúde da Família (ESF) e, posteriormente, com o intuito de reorganizar o SUS, com a Política Nacional de Atenção Básica (PNAB), as ESF se tornaram o principal meio de expansão, consolidação e qualificação da APS. ${ }^{2}$

Paralelamente, há um movimento de busca pela melhoria da qualidade assistencial prestada aos usuários dos serviços de saúde, monitorada por estratégias de avaliação de serviços, como os sistemas de acreditação, utilizados em mais de 70 países, processo que é administrado por governos ou por organismos de avaliação independentes. ${ }^{3} \mathrm{~A}$ acreditação refere-se ao reconhecimento do cumprimento de modelos pré-estabelecidos e de referência e excelência em saúde, que devem ser realizados por meio de avaliadores, em pares, externos. Em nível de APS, Estados Unidos, Canadá, Austrália, Reino Unido, Nova Zelândia, Jordânia, Arábia Saudita, Líbano e Egito possuem modelos de acreditação bem desenvolvidos e de alta qualidade. Estes modelos constituem-se em ferramentas úteis para melhorar a segurança e a qualidade dos serviços de saúde, com destaque para os da atenção primária. ${ }^{4}$

No Brasil, o Ministério da Saúde introduziu o Programa Nacional de Melhoria do Acesso e da Qualidade da Atenção Básica (PMAQ-AB) para incentivar gestores e equipes a melhorar a qualificação das ações ofertadas à população do território, com incentivo financeiro conforme a adesão. O programa recomenda ações estratégicas com o objetivo de melhorar os resultados das ações da APS, considerando um padrão de qualidade uniforme, possibilitando maior efetividade das ações governamentais destinadas a esse nível de atenção. ${ }^{5}$

Nessa perspectiva, no Estado do Paraná definiu-se como ação estratégica a implantação do Programa de Qualificação da Atenção Primária à Saúde, o APSUS, um programa de apoio aos municípios, que propõe a melhoria da APS em nível estadual, sendo referência para os demais estados brasileiros. O programa é dividido em três componentes principais: investimento na melhoria da estrutura da unidade, custeio para a equipe, com incentivo estadual mensal, e educação permanente. ${ }^{6}$ Em razão disso, a Secretaria de Estado da Saúde elaborou uma metodologia denominada de "Tutoria" que promove a aplicação de conceitos dos princípios da qualidade, bem como dos atributos e funções da APS na realidade de cada equipe de saúde. Assim, têm-se o estabelecimento de padrões e protocolos, organização do processo de trabalho, garantia de segurança do usuário e do profissional que, consequentemente, geram melhoria da atenção, dos indicadores de saúde e da satisfação do usuário. ${ }^{6} \mathrm{O}$ processo da avaliação reflete-se nos selos hierárquicos de certificação: bronze (gerenciamento de risco), prata (gerenciamento do processo), ouro (garantia de resultados a população) e diamante (consolidação do processo de trabalho, com excelência na gestão), que são atribuídos às equipes de saúde que atingiram os padrões de qualidade esperados. ${ }^{7}$

Como protagonistas do desenvolvimento e melhoria desse sistema de saúde, estão os trabalhadores da APS, que desenvolvem suas atividades laborais em um ambiente nem sempre favorável às suas práticas profissionais, ${ }^{8}$ manifesto por pessoal da saúde, equipamentos e insumos insuficientes, estrutura física inadequada, remuneração baixa e exposição a riscos à saúde biopsicossocial e estes fatores favorecem o desenvolvimento do estresse ocupacional., 9,10

O estresse ocupacional provém do ambiente laboral e envolve aspectos da organização, da gestão, das condições e da qualidade das relações interpessoais no trabalho. ${ }^{11,12}$ Entre os modelos teóricos mais adotados para avaliação de estresse no trabalho, está o Demanda - Controle - Apoio Social, proposto por Theorell e Karasek, que compreende o estresse ocupacional como resultado da interação entre muitas demandas psicológicas provenientes do ambiente de trabalho, menor controle sobre o processo laboral e menor apoio social recebido de colegas e chefes. $^{12}$

As demandas se referem às exigências psicológicas do trabalho, envolvendo tempo, velocidade e intensidade na realização das atividades, e as demandas conflitantes; o controle, ao uso de suas habilidades intelectuais e a autonomia para a tomada de decisão sobre o seu próprio trabalho; e o apoio social se refere a interação e suporte oferecido nas relações interpessoais no trabalho, que podem minimizar os eventos estressantes, pois colabora na superação das dificuldades, promove bem-estar e satisfação no ambiente de trabalho. ${ }^{12}$

Desse modo, entende-se que a organização do trabalho da equipe saúde da família (eSF) afeta a saúde de quem nela trabalha, podendo o modelo gerencial de qualidade influenciar na demanda, controle e apoio social no trabalho, em decorrência das exigências constituídas nos modelos de avaliação e acreditação do serviço, em que os profissionais estão inseridos. Altos níveis de estresse ocupacional predispõem ao adoecimento dos trabalhadores e aumentam o presenteísmo, absenteísmo, rotatividade, insatisfação laboral, eventos adversos e, por consequência, repercutem na assistência prestada à população. ${ }^{13}$

Pelos motivos expostos e a incipiência de estudos publicados que comparem o estresse ocupacional em organizações de cuidados primários certificadas e não certificadas, faz-se importante o desenvolvimento de pesquisas que realizem essa análise..$^{9,14}$ Tais resultados poderão subsidiar ações para melhoria das condições de trabalho e na qualidade de assistência prestada à comunidade.

Assim, este estudo objetivou comparar o estresse ocupacional em trabalhadores de equipes saúde da família certificadas e não certificadas com selo de qualidade assistencial pela Tutoria da Atenção Primária à Saúde. Defende-se a hipótese de que os 
profissionais que atuam nos serviços certificados com o selo de qualidade apresentam maior demanda psicológica, controle e apoio social no trabalho em relação aqueles de equipes não certificadas.

\section{MÉTODOS}

Estudo transversal desenvolvido com trabalhadores das equipes de ESF de dois municípios do estado do Paraná, denominados de A e B. O município A apresenta, aproximadamente, $65 \mathrm{mil}$ habitantes, densidade demográfica de $126 \mathrm{hab} / \mathrm{km}^{2}, 9$ unidades básicas de saúde (UBS), com 15 equipes de ESF, todas elas certificadas com Selo Bronze de qualidade em APS pela Tutoria há um ano. $\mathrm{O}$ município $\mathrm{B}$ possui, aproximadamente, 32 mil habitantes, 72 hab/ $\mathrm{km}^{2}$, 8 UBS e 8 equipes de ESF, as quais não possuem qualquer tipo de certificação de qualidade. Esses municípios foram escolhidos por intencionalidade, visto que $94,9 \%$ das cidades brasileiras se classificam como pequeno e médio porte e apresentam similaridades na gestão em saúde. ${ }^{15}$

O processo de avaliação da Tutoria visa qualificar a APS, por meio da adesão voluntária pelas eSF e gestores municipais, tendo a premissa de que os resultados alcançados dependem do envolvimento de todos os atores. Dentre as diretrizes, ressaltam-se a definição dos parâmetros de qualidade, a partir da análise das diferentes realidades de saúde; além do estímulo contínuo aos padrões de melhoria do acesso e da qualidade; mobilização dos gestores municipais e estaduais para a qualificação da atenção primária e fortalecimento dos modelos de atenção previstos na PNAB. ${ }^{7}$ Diferente do PMAQ, trata-se de um modelo assistencial de qualidade sem incentivo financeiro por adesão, razão que motivou a escolha da Tutoria na análise deste estudo.

Até o ano de 2018, 358 municípios aderiram ao processo de avaliação da Tutoria, totalizando 1.070 UBS, das quais 193 foram certificadas, sendo 165 com Selo Bronze, 28 com Selo Prata e 03 com Selo Ouro. O Selo Bronze, primeiro nível de qualidade, alcançado por todas as UBS do município $A$, reúne itens que visam gerenciamento dos riscos com foco na segurança do cidadão e da equipe. O processo de certificação ocorre por meio da aplicação do um Instrumento para Avaliação da Qualidade na Atenção Primária à Saúde, composto por 105 itens, categorizados em dois grandes eixos de análise, sendo Gestão da Unidade e Atributos da APS. No primeiro eixo, são contempladas questões referentes à infraestrutura, recursos humanos, recursos materiais e tecnológicos, gerenciamento de riscos, entre outros. Já no segundo, abordam-se itens relacionados ao primeiro contato do paciente, longitudinalidade, integralidade, coordenação, focalização na família, orientação comunitária e competência cultural. $^{7}$

Anteriormente à avaliação ocorre a elaboração de protocolos, readequação de fluxos e processos, bem como da estrutura física e outros aspectos, para os quais há o envolvimento da equipe gestora e assistencial das eSF. No momento da avaliação estas unidades recebem profissionais pertencentes a outras regionais de saúde para aplicação do instrumento, encontradas inconformidades o gestor possui um prazo de 90 dias para correções e somente após as adequações e reavaliação a unidade alcança a certificação. ${ }^{7}$

$\mathrm{Na}$ ocasião do levantamento dos possíveis participantes da pesquisa, identificaram-se 286 trabalhadores, sendo 153 do município com eSF certificadas e 104 do município com eSF não certificadas. Para o cálculo do tamanho amostral, fixou-se proporção de $50 \%$, visto que esse valor implica tamanho máximo de amostra, o nível de significância de 5\% $(\mathrm{Za}=0,05)$ e erro amostral absoluto de 5\% (E=0,05), resultando em um mínimo de 164 profissionais.

Os critérios de inclusão foram pertencer à equipe da ESF e trabalhar no local de estudo há, no mínimo, um ano. Os critérios de exclusão foram estar afastado do trabalho por licenças ou férias, não estar presente no local de trabalho no período da coleta de dados e falta de resposta em mais de $20 \%$ do questionário.

Entre janeiro e março de 2019, convidou-se todos os elegíveis nas ESF em horários de trabalho que não estavam em atendimento e previamente acordados com a coordenação, e os esclareceu sobre o objetivo da pesquisa. Os questionários foram entregues aos que consentiram e após respondidos eram devolvidos aos pesquisadores, totalizando 178 participantes, dos quais 106 (69,3\%) pertenciam ao município com equipes de ESF certificadas e 72 (69,2\%) de equipes não certificadas.

Para obtenção dos dados, elaborou-se um questionário com variáveis sociodemográficas: sexo (feminino $x$ masculino), idade (em anos) e situação conjugal (sem $x$ com relacionamento conjugal); e ocupacionais: cargo/função ocupada (médico, enfermeiro, odontólogo, técnico/auxiliar de enfermagem, técnico em saúde bucal, agente comunitário de saúde e agente de endemias), outro vínculo empregatício (não x sim), anos de trabalho na ESF em estudo (em anos) e remuneração suficiente para satisfazer as necessidades (não x sim).

Para avaliar o estresse ocupacional utilizou-se a Job Stress Scale, em sua versão traduzida e validada para o português do Brasil. ${ }^{16}$ Trata-se de um instrumento autoaplicável, composto por 17 itens, que avaliam três dimensões conceituais: demanda, controle e apoio social. As respostas são fornecidas em escalas do tipo Likert de 1 a 4, variando de menor a maior intensidade. A pontuação das dimensões demanda e o controle são dicotomizadas em alto e baixo, utilizando a mediana como ponto de corte, permitindo a definição de quatro tipos de trabalho: job strain ou trabalho de alto desgaste (alta demanda e baixo controle), trabalho ativo (alta demanda e alto controle), trabalho passivo (baixa demanda e baixo controle) e trabalho de baixo desgaste (baixa demanda e alto controle). ${ }^{12,16}$

A análise de dados foi realizada no programa Statistical Package for the Social Sciences (SPSS), versão 20.0, por análises descritivas e inferenciais. A comparação das características sociodemográficas e ocupacionais dos trabalhadores de saúde foi realizada pelo teste exato de Fisher. A regressão logística univariada foi usada para comparar demanda, controle, apoio social e os tipos de trabalho entre os trabalhadores submetidos aos diferentes modelos gerenciais. Posteriormente, por meio de regressão logística múltipla, sexo, idade, anos de profissão, 
nível de escolaridade do cargo e suficiência de recursos financeiros para as necessidades foram considerados como potenciais confundidores e ajustaram a relação. Os resultados foram apresentados em odds ratio (OR) brutos e ajustados com intervalos de confiança de $95 \%$, ou seja, em todas as análises considerou-se $p<0,05$ como estatisticamente significativo.

Este estudo seguiu as recomendações de ética em pesquisa envolvendo seres humanos, sendo aprovado por Comitê de Ética em Pesquisa conforme Parecer $n^{\circ}$.2.302.907. Todos os participantes assinaram o Termo de Consentimento Livre e Esclarecido.

\section{RESULTADOS}

A amostra desta pesquisa foi composta por 178 trabalhadores, dos quais $106(69,3 \%)$ pertenciam ao município com equipes certificadas e $72(69,2 \%)$ ao município com equipes não certificadas pela Tutoria da Atenção Primária. Participaram do estudo 11 médicos, 11 odontólogos, 24 enfermeiros, 46 técnicos/ auxiliares de enfermagem, 12 auxiliares de odontologia e 73 agentes comunitários de saúde/agentes de endemia (ACS/ACE).
Ao se comparar as características sociodemográficas e ocupacionais, verificou-se, na Tabela 1, que se trata de pessoas com características semelhantes com exceção da situação conjugal e remuneração, pois a maioria dos profissionais vinculados a equipes certificadas possuía relacionamento conjugal estável e recursos financeiros suficientes para satisfazer as necessidades.

Ao associar essas características sociodemográficas e ocupacionais com as dimensões do estresse ocupacional, o baixo controle sobre o trabalho esteve associado ao exercício de cargos de nível médio $(p<0,001)$ nas equipes não certificadas. $O$ baixo apoio social associou-se a percepção de remuneração insuficiente para as necessidades entre os trabalhadores de eSF certificadas $(p=0,014)$. As demais variáveis não apresentaram associação estatisticamente significativa.

A comparação das dimensões da Job Stress Scale entre os trabalhadores das eSF certificadas e não certificadas pela Tutoria da APS estão apresentadas na Tabela 2. Verificouse que os trabalhadores de eSF certificadas apresentaram 4,164 chances de alta demanda psicológica em relação àqueles de equipes não certificadas. Apesar do baixo

Tabela 1. Comparação das variáveis sociodemográficas e ocupacionais em trabalhadores de Estratégias Saúde da Família certificadas e não certificadas pela Tutoria da Atenção Primária da Saúde no norte do Paraná, Brasil, 2019 (n=178).

\begin{tabular}{|c|c|c|c|}
\hline Variável & Não certificada pela Tutoria & Certificada pela Tutoria & $p$-value* \\
\hline \multicolumn{4}{|l|}{ Sexo } \\
\hline Masculino & $12(16,7 \%)$ & $10(9,4 \%)$ & \multirow[t]{2}{*}{0,114} \\
\hline Feminino & $60(83,3 \%)$ & $96(90,6 \%)$ & \\
\hline \multicolumn{3}{|l|}{ Idade } & \multirow{3}{*}{0,424} \\
\hline $22-40$ anos & $44(61,1 \%)$ & $62(58,5 \%)$ & \\
\hline $41-70$ anos & $28(38,9 \%)$ & $44(41,5 \%)$ & \\
\hline \multicolumn{3}{|l|}{ Situação conjugal } & \multirow{3}{*}{0,001} \\
\hline Sem relação conjugal & $33(45,8 \%)$ & $23(21,7 \%)$ & \\
\hline Com relação conjugal & $39(54,2 \%)$ & $83(78,3 \%)$ & \\
\hline \multicolumn{3}{|l|}{ Outro vínculo empregatício } & \multirow{3}{*}{0,211} \\
\hline Não possui & $60(83,3 \%)$ & $94(88,7 \%)$ & \\
\hline Possui & $12(16,7 \%)$ & $12(11,3 \%)$ & \\
\hline \multicolumn{3}{|l|}{ Nível do cargo } & \multirow{3}{*}{0,194} \\
\hline Ensino médio/pós-médio & $50(69,4 \%)$ & $81(76,4 \%)$ & \\
\hline Nível superior & $22(30,6 \%)$ & $25(23,6 \%)$ & \\
\hline \multicolumn{3}{|l|}{ Tempo de trabalho na unidade } & \multirow{3}{*}{0,300} \\
\hline $1-5$ anos & $37(51,4 \%)$ & $49(46,2 \%)$ & \\
\hline 6-33 anos & $35(48,6 \%)$ & $57(53,8 \%)$ & \\
\hline \multicolumn{3}{|c|}{ Remuneração suficiente para as necessidades } & \multirow{3}{*}{0,001} \\
\hline Não & $58(80,6 \%)$ & $62(58,5 \%)$ & \\
\hline Sim & $14(19,4 \%)$ & $44(41,5 \%)$ & \\
\hline
\end{tabular}

*Teste exato de Fisher

Fonte: Dados da pesquisa, 2019. 
Tabela 2. Comparação da demanda, controle e apoio social em trabalhadores de Estratégias Saúde da Família certificadas e não certificadas pela Tutoria da Atenção Primária da Saúde no norte do Paraná, Brasil, 2019 (n=178).

\begin{tabular}{|c|c|c|c|c|c|c|}
\hline Dimensão & $\begin{array}{c}\text { Não certificada } \\
\text { pela Tutoria }\end{array}$ & $\begin{array}{l}\text { Certificada } \\
\text { pela Tutoria }\end{array}$ & $p$-value & $\begin{array}{l}\text { Odds Ratio bruto } \\
\text { (Intervalo de Confiança 95\%) }\end{array}$ & $p$-value & $\begin{array}{c}\text { Odds Ratio ajustado* } \\
\text { (Intervalo de Confiança 95\%) }\end{array}$ \\
\hline \multicolumn{7}{|l|}{ Demanda } \\
\hline Baixa & $53(73,6 \%)$ & $45(42,5 \%)$ & & 1 & & 1 \\
\hline Alta & $19(26,4 \%)$ & $61(57,5 \%)$ & $<0,001$ & $3,781(1,973-7,246)$ & $<0,001$ & $4,164(2,102-8,249)$ \\
\hline \multicolumn{7}{|l|}{ Controle } \\
\hline Baixo & $46(63,9 \%)$ & $67(63,2 \%)$ & 0,926 & $1,030(0,553-1,919)$ & 0,891 & $1,047(0,542-2,022)$ \\
\hline Alto & $26(36,1 \%)$ & $39(36,8 \%)$ & & 1 & & 1 \\
\hline \multicolumn{7}{|l|}{ Apoio social } \\
\hline Baixo & $38(52,8 \%)$ & $72(67,9 \%)$ & 0,042 & $1,895(1,023-3,511)$ & 0,048 & $1,896(1,006-3,573)$ \\
\hline Alto & $34(47,2 \%)$ & $34(32,1 \%)$ & & 1 & & 1 \\
\hline
\end{tabular}

*ajustado por sexo, idade, anos de trabalho, nível de escolaridade do cargo e recursos financeiros suficientes

Fonte: Dados da pesquisa, 2019.

Tabela 3. Comparação dos tipos de trabalhos em trabalhadores de Estratégias Saúde da Família certificadas e não certificadas pela Tutoria da Atenção Primária da Saúde no norte do Paraná, Brasil, 2019 (n=178).

\begin{tabular}{|c|c|c|c|c|c|c|}
\hline Tipo de trabalho & $\begin{array}{l}\text { Não certificada } \\
\text { pela Tutoria }\end{array}$ & $\begin{array}{l}\text { Certificada } \\
\text { pela Tutoria }\end{array}$ & $p$-value & $\begin{array}{c}\text { Odds Ratio bruto } \\
\text { (Intervalo de Confiança 95\%) }\end{array}$ & $p$-value & $\begin{array}{c}\text { Odds Ratio } \\
\text { (Intervadado* }\end{array}$ \\
\hline \multicolumn{7}{|l|}{ Job strain } \\
\hline Não & $64(88,9 \%)$ & $69(65,1 \%)$ & & 1 & & 1 \\
\hline Sim & $8(11,1 \%)$ & $37(34,9 \%)$ & 0,001 & $4,290(1,859-9,902)$ & $<0,001$ & $4,956(2,067-11,882)$ \\
\hline \multicolumn{7}{|l|}{ Trabalho passivo } \\
\hline Não & $34(47,2 \%)$ & $76(71,7 \%)$ & & 1 & & 1 \\
\hline Sim & $38(52,8 \%)$ & $30(28,3 \%)$ & 0,001 & $0,353(0,189-0,661)$ & $<0,001$ & $0,293(0,148-0,580)$ \\
\hline \multicolumn{7}{|l|}{ Trabalho ativo } \\
\hline Não & $61(84,7 \%)$ & $82(77,4 \%)$ & & 1 & & 1 \\
\hline Sim & $11(15,3 \%)$ & $24(22,6 \%)$ & 0,228 & $1,623(0,739-3,565)$ & 0,251 & $1,623(0,710-3,711)$ \\
\hline \multicolumn{7}{|l|}{ Baixo desgaste } \\
\hline Não & $57(79,2 \%)$ & $91(85,8 \%)$ & & 1 & & 1 \\
\hline Sim & $15(20,8 \%)$ & $15(14,2 \%)$ & 0,245 & $0,626(0,285-1,378)$ & 0,297 & $0,651(0,291-1,459)$ \\
\hline
\end{tabular}

*ajustado por sexo, idade, anos de trabalho, nível de escolaridade do cargo e recursos financeiros suficientes

Fonte: Dados da pesquisa, 2019.

controle sobre o trabalho ser menor entre os participantes de equipes certificadas, não houve diferença estatisticamente significativa. As chances de baixo apoio social no trabalho foram significativamente maiores entre os profissionais das eSF certificadas, quando comparados aqueles de unidades não certificadas.

A presença de job strain foi significativamente maior entre os trabalhadores de eSF certificadas. Por outro lado, o trabalho passivo foi menor, indicando que entre os trabalhadores de unidades não certificadas esse tipo de trabalho foi mais prevalente. Essa relação permaneceu significativa mesmo levando em consideração sexo, idade, anos de profissão, nível de escolaridade do cargo e suficiência de recursos financeiros para as necessidades (Tabela 3).

Ao comparar os tipos de trabalho segundo as categorias profissionais, observou-se que entre aqueles vinculados a ESF certificadas predominou o job strain nos enfermeiros (46,2\%), nos técnicos e auxiliares de enfermagem $(40,0 \%)$ e nos ACS/ACE (29,5\%); o trabalho passivo nos médicos $(40,0 \%)$ e nos auxiliares de odontologia $(57,1 \%)$; e o trabalho ativo nos odontólogos $(57,1 \%)$. Por outro lado, a maioria dos odontólogos $(50,0 \%)$, médicos $(50,0 \%)$ e enfermeiros $(30,0 \%)$ que trabalhavam em unidades não certificadas apresentou trabalho de baixo desgaste; e entre os técnicos e auxiliares de enfermagem (62,5\%), os ACS/ACE 
$(69,0 \%)$ e os auxiliares de odontologia $(60,0 \%)$ predominou o trabalho passivo.

\section{DISCUSSÃO}

No presente estudo comparou-se o estresse ocupacional entre trabalhadores de eSF que possuem e que não possuem certificação de qualidade em APS da Tutoria. Os resultados indicaram que o estresse ocupacional apresentou maior prevalência em profissionais de equipes certificadas com o Selo Bronze de qualidade, pois eles apresentaram maior percepção de demanda psicológica de trabalho e baixo apoio social, além de predominância de job strain.

O processo de trabalho em saúde é resultado de um conjunto de ações coordenadas desenvolvidas pelos trabalhadores, em que indivíduos, famílias e grupos sociais compõe o objeto de trabalho, e saberes e métodos representam os instrumentos que originam a atenção em saúde. ${ }^{17,18}$

A demanda da APS é composta por atributos e funções que a tornam complexa, principalmente por ser a coordenadora das Redes de Atenção à Saúde $\mathrm{e}^{6,19} \mathrm{e}$ o meio prioritário para cobertura universal de saúde. ${ }^{20}$ Nesse sentido, a Agenda 2030 da Organização das Nações Unidas, por meio do Objetivo de Desenvolvimento Sustentável - ODS 3 propõe o alcance da cobertura universal de saúde, incluindo acesso a serviços essenciais de saúde de qualidade e a medicamentos e vacinas essenciais seguros, eficazes, de qualidade e acessíveis para todos. ${ }^{21}$ Os processos de certificação de qualidade têm potencial de orientar esse caminho e fornecer uma estrutura facilitadora para a prestação de assistência de alta qualidade ao usuário. ${ }^{22}$

A certificação de qualidade na APS além de fortalecer o controle e a melhoria da qualidade, promove mudanças relacionais e estratégicas nas organizações, ademais de avanços significativos em seu desempenho. Ainda, produz gestores com foco nos valores da qualidade, aumenta o comprometimento das equipes de saúde com a política organizacional, e aprimora o trabalho em equipe, o acesso e a qualidade do atendimento, e a segurança do paciente. ${ }^{22}$

O Selo Bronze prima pela segurança do usuário e da equipe de saúde, por meio de dois eixos: gestão e atributos da APS. O instrumento de avaliação contém 105 itens a serem alcançados e a sua forma de verificação, dos quais destacam-se: a identificação da unidade, a estrutura física e de recursos materiais para atendimento adequado à população, a acessibilidade, a biossegurança e a estratificação de risco e atendimentos conforme linhas guias Rede Mãe Paranaense, Hipertensão, Diabetes, Infarto, Saúde Mental, Saúde Bucal e Saúde do Idoso.23

O processo gerencial proposto pela Tutoria objetiva ser um exercício diário e constante de aprendizagem, proporcionando a conexão da teoria com a prática, desenvolvimento de competências técnicas, comunicacionais e relacionais, com foco na melhoria da qualidade técnica e humanística no cotidiano dos serviços. Além disso, a Tutoria prevê a criação de um clima de apoio e suporte a equipe. ${ }^{6}$ No entanto, como ocorre em outros países, ${ }^{3} a$ preocupação com a qualidade em conformidade com saúde e segurança do trabalhador é limitada aos riscos ocupacionais tradicionais, especificamente, os biológicos. ${ }^{23}$

Promover qualidade assistencial na saúde por meio de processos gerenciais de certificação de qualidade foi indicado por estudo com enfermeiros de três instituições hospitalares do Estado de São Paulo, como um fator que repercutiu em maiores níveis de estresse ocupacional e impactou negativamente na saúde mental dos pesquisados devido a aspectos relacionais e à complexidade do trabalho, ${ }^{24}$ corroborando com os achados deste estudo. Quando os recursos necessários ao trabalho e as demandas da função não se encontram ajustadas ao trabalhador, ocorrem respostas físicas e emocionais levam ao estresse, e a insuficiência de recursos psicológicos preexistentes conduz ao adoecimento biopsicosocial. ${ }^{12,16}$

As eSF certificadas pelo selo de qualidade apresentaram chances significativamente maiores de alta demanda psicológica em relação àqueles de equipes não certificadas. Considerando que a demanda reflete as pressões psicológicas quantitativas e qualitativas do trabalho, ${ }^{12}$ e que o Selo Bronze requer uma padronização dos processos de gestão da unidade e dos atributos da APS, infere-se que o cumprimento dos 105 itens de verificação ${ }^{23}$ implica em criar hábitos, comportamentos e estratégias para o alcance das metas de qualidade dentro de um cronograma estabelecido, o que resulta em execução das atividades laborais com rapidez e intensidade em curtos prazos, aumentando a demanda psicológica, em relação aqueles que lidam apenas com as demandas psicológicas já existentes e comuns ao processo de trabalho na APS.

Ainda, esse modelo de gestão pautado em um método avaliativo de produção de trabalho com metas de qualidade, requer que cada membro da equipe esteja preparado e disposto a realizar o pactuado, ${ }^{25}$ pois na APS a prática deve ser colaborativa e interprofissional para alcançar adequadamente os resultados de saúde desejados, ${ }^{26}$ o que pode não estar ocorrendo, pois o trabalho em equipe é uma fragilidade a ser trabalhada nos serviços de saúde, ${ }^{27}$ e a percepção de apoio social dos pares foi menor entre os profissionais de unidades de certificadas.

$\mathrm{O}$ alto controle sobre o trabalho foi maior entre os profissionais de ESF certificadas, todavia essa não foi uma diferença estatisticamente significativa. Assim, a possibilidade do trabalhador de utilizar suas habilidades intelectuais para a realização do seu trabalho, bem como decidir de que forma irá realizá-lo, ${ }^{12}$ é semelhante entre os profissionais de eSF certificadas ou não. A ausência de diferença na autonomia reportada pelos trabalhadores em processos gerenciais díspares pode estar relacionada ao fato da estabilidade conferida no serviço público e da independência na realização dos protocolos e na assistência prestada às pessoas. ${ }^{28}$

A coexistência de altas demandas psicológicas com baixo controle sobre o trabalho é denominada de job strain, ${ }^{12}$ cuja prevalência foi maior nas ESF certificadas, indicando que essas unidades, quando comparadas as não certificadas, possuem maior chance de apresentar profissionais com alto desgaste, baixa autonomia nas tomadas de decisão, com pouco uso da sua 
criatividade no processo de trabalho em saúde. Esses aspectos geram desmotivação e possuem maior probabilidade de efeitos nocivos à saúde, como o desenvolvimento de depressão. ${ }^{29}$

Ainda, a predominância desse tipo de trabalho foi maior na equipe de enfermagem, pode estar relacionado ao fato desses profissionais atuarem em todos os processos e procedimentos das ESF. Os enfermeiros, ainda, são os responsáveis pelo gerenciamento das unidade ${ }^{30} \mathrm{e}$, por essa razão, são os responsáveis pelo cumprimento das metas de qualidade pactuadas e sofrem diretamente maior pressão dos gestores para o cumprimento das exigências estabelecidas. Pelo maior risco de adoecimento e desgaste, ações organizacionais que melhoram as habilidades dos enfermeiros são eficazes no desenvolvimento de mecanismos de enfrentamento ao estresse. ${ }^{31}$

O trabalho passivo também predispõe ao adoecimento, visto que, devido à baixa demanda psicológica e baixo controle, o trabalho é repetitivo, monótono, com poucas oportunidades de novos aprendizados, tornando o trabalhador apático e ocorre o declínio do seu desenvolvimento laboral. ${ }^{12}$ Esse tipo de trabalho foi predominante nas eSF não certificadas, sobretudo entre técnicos e auxiliares de enfermagem, ACS/ACE e os auxiliares de odontologia.

Neste estudo, a proporção de trabalho ativo foi semelhante nas eSF certificadas e não certificadas. Nesse tipo de trabalho apesar das pessoas apresentarem alta demanda psicológica para execução de seu trabalho, possuem autonomia na tomada de decisões e utilizam seu potencial intelectual para o cumprimento do processo de trabalho em saúde. ${ }^{11}$

A situação de trabalho considerada ideal é aquela em que há baixas demandas psicológicas e alto controle sobre o trabalho realizado, o trabalho de baixo desgaste, ${ }^{12}$ que também não apresentou diferenças estatísticas nas proporções de trabalhadores das unidades certificadas e não certificadas com selo de qualidade.

As chances de alto apoio social no trabalho foram significativamente menores no pessoal de saúde das eSF certificadas, quando comparados aqueles de unidades não certificadas. $O$ apoio social refere-se ao apoio recebido pelo trabalhador, de seus colegas de trabalho e supervisores, refletindo, portanto, as relações interpessoais no trabalho, que é um fator decisivo nas vivências de satisfação, insatisfação e êxito no trabalho, com potencial de transformar o sofrimento em prazer. ${ }^{32}$ Diante do exposto, o apoio social deve permear todo o processo de certificação das equipes das unidades de saúde, visto que as metas e desafios são coletivos e em prol do acesso universal de qualidade a população do território. A percepção de remuneração insuficiente foi associada ao baixo apoio social, inferindo-se que na percepção dos trabalhadores as atividades desempenhadas podem ser reconhecidas pelos gestores por meio de pró-labore compatível ao seu empenho e qualificação para o processo de certificação.

O movimento da gestão da qualidade é importante e a sua adesão pelas equipes de saúde no âmbito da APS deve ser incentivada como ação proativa e voluntária do serviço de saúde, em busca da qualidade e excelência no serviço prestado. No entanto, as metas, muitas vezes, não contemplam aspectos relacionados à saúde do trabalhador, que são os protagonistas desse processo. Por isso, se faz necessário a inclusão desses fatores na avaliação de qualidade, bem como é importante que os gestores municipais busquem estratégias para gerenciar o estresse ocupacional, prevenindo o adoecimento mental.

Corrobora com esse pensamento, um estudo realizado no Líbano, no qual os autores refletem que os altos padrões de saúde e segurança do trabalhador de saúde não se constituem em estratégia de marketing para quem recebe atendimento e há pouco incentivo de mercado para melhorar esses padrões, razões para os administradores dessas instituições não investirem em programas de promoção à saúde ocupacional. Sem esses incentivos, as agências reguladoras devem incentivar arranjos justos de trabalho e condições de trabalho seguras e saudáveis para a força de trabalho em saúde. ${ }^{3}$ Os sistemas de avaliação de qualidade podem propor incentivos para melhorar a qualidade da saúde e segurança do trabalhador, tais como: inserir itens de avaliação relativos a serviço de apoio a saúde física e psicológica do trabalhador; cumprimento da legislação vigente, principalmente da Norma Regulamentadora 32; recursos financeiros exclusivos pelos serviços concluídos relativos a saúde dos trabalhadores, entre outros.

\section{CONCLUSÃO E IMPLICAÇÕES PARA A PRÁTICA}

Os trabalhadores pertencentes às equipes de ESF com certificação de qualidade na prestação de seus serviços apresentaram maiores chances de estresse ocupacional em relação aqueles vinculados as unidades não certificadas.

Acredita-se que os achados dessa pesquisa possam embasar mudanças na implantação desses modelos gerenciais de qualidade da assistência de forma a considerar a saúde dos trabalhadores envolvidos, sobretudo: programas de apoio para lidar com as demandas psicológicas do trabalho; promoção de um clima organizacional que incentive o apoio social e a colaboração interprofissional em saúde; reuniões periódicas para identificar as dificuldades dos profissionais na implementação das ações, traçando um planejamento estratégico de superação; e reconhecimento do trabalho realizado.

A principal limitação deste estudo é a impossibilidade de generalização dos resultados, tanto pelo delineamento transversal, quanto pela amostra de profissionais pertencentes a equipes certificadas com apenas um tipo de selo assistencial e restrita a uma única região de um estado brasileiro. Adicionalmente, há o viés de conveniência social, caracterizado pela presença dos gestores das unidades de saúde e preenchimento do instrumento de pesquisa no local de trabalho, pois os entrevistados podem ter fornecido respostas que consideraram desejáveis e socialmente aceitas. No entanto, os resultados não devem ser subestimados, pois os trabalhadores foram informados sobre a confidencialidade dos dados e o anonimato. Este estudo não 
avaliou a influência das cargas de trabalho dos profissionais no estresse ocupacional e esse aspecto pode ser um determinante, assim futuros estudos devem explorar essa relação nos diferentes processos gerenciais da APS, bem como os fatores associados ao estresse entre as eSF certificadas.

Por outro lado, este estudo apresenta fortalezas por ter sido realizado em cidades de pequeno e médio porte, representando a maioria dos municípios brasileiros. Ainda, deve-se considerar o ineditismo ao analisar o estresse ocupacional em trabalhadores submetidos a diferentes modelos de gestão e incluir todas as categorias profissionais da eSF.

Os resultados foram apresentados para os profissionais e gestores das ESF, em reunião específica nos municípios estudados, o que propicia aos gestores e os trabalhadores a elaboração de estratégias conjuntas para minimizar o estresse, fundamental para a manutenção da saúde e do processo de qualidade para a melhoria dos serviços.

\section{CONTRIBUIÇÕES DOS AUTORES}

Desenho do estudo. Luiza Ferreira Rigonatti Silva. Alessandro Rolim Scholze. Maria José Quina Galdino.

Coleta ou produção dos dados. Luiza Ferreira Rigonatti Silva. Análise de dados. Luiza Ferreira Rigonatti Silva. Maria José Quina Galdino.

Interpretação dos resultados. Alessandro Rolim Scholze. Paloma de Souza Cavalcante Pissinati. Janaína Recanello Begui. Maynara Fernanda Carvalho Barreto.

Redação e revisão crítica do manuscrito. Luiza Ferreira Rigonatti Silva. Alessandro Rolim Scholze. Paloma de Souza Cavalcante Pissinati. Janaína Recanello Begui. Maynara Fernanda Carvalho Barreto. Maria José Quina Galdino.

Aprovação da versão final do artigo. Luiza Ferreira Rigonatti Silva. Alessandro Rolim Scholze. Paloma de Souza Cavalcante Pissinati. Janaína Recanello Begui. Maynara Fernanda Carvalho Barreto. Maria José Quina Galdino.

Responsabilidade por todos os aspectos do conteúdo e a integridade do artigo. Luiza Ferreira Rigonatti Silva. Alessandro Rolim Scholze. Paloma de Souza Cavalcante Pissinati. Janaína Recanello Begui. Maynara Fernanda Carvalho Barreto. Maria José Quina Galdino.

\section{EDITOR ASSOCIADO}

\author{
Gerson Luiz Marinho
}

\section{REFERÊNCIAS}

1. Giovanella L, Almeida PF. Comprehensive primary care and segmented health systems in South America. Cad Saude Publica. 2017;33(33, Suppl 2):e00118816. http://dx.doi.org/10.1590/0102-311x00118816. PMid:28977122.

2. Portaria ํㅡ 2.436, de 21 de setembro de 2017 (BR). Aprova a Política Nacional de Atenção Básica, estabelecendo a revisão de diretrizes para a organização da Atenção Básica, no âmbito do Sistema Único de Saúde (SUS). Diário Oficial da União, Brasília (DF), 22 set 2017.
3. Habib RR, Blanche G, Souha F, El-Jardali F, Nuwayhid I. Occupational health and safety in hospitals accreditation system: the case of Lebanon. Int J Occup Environ Health. 2016;22(3):201-8. http://dx.doi.org/10.108 0/10773525.2016.1200211. PMid:27398975.

4. Tabrizi J, Gharibi F. Primary healthcare accreditation standards: a systematic review. Int J Health Care Qual Assur. 2019;32(2):310-20. http://dx.doi.org/10.1108/IJHCQA-02-2018-0052. PMid:31017069.

5. Mota RRA, David HMSL. National primary care access and quality improvement program: issues to discuss. Rev Enferm UERJ. 2015;23(1):122-7. http://dx.doi.org/10.12957/reuerj.2015.14725.

6. Secretaria de Estado da Saúde do Paraná. A Tutoria na APS [Internet] Curitiba, PR; 2017. [citado 2020 out 15]. Disponível em: http://www. saude.pr.gov.br/arquivos/File/apostila_apsus_final.pdf

7. Secretaria de Estado da Saúde do Paraná. Resolução SESA n 741/2018 Instituir e regulamentar a Tutoria como Processo de Qualidade na Atenção Primária em Saúde (APS) [Internet]. Curitiba, PR;2018. [citado 2020 out 15]. Disponível em: http://www.saude.pr.gov.br/arquivos/File/-- Resolucoes2018/741_18.PDF

8. Biff D, Pires DEP, Forte ECN, Trindade LL, Machado RR, Amadigi FR et al. Nurses' workload: lights and shadows in the Family Health Strategy. Cien Saude Colet. 2020;25(1):147-58. http://dx.doi.org/10.1590/141381232020251.28622019. PMid:31859863.

9. Leonelli LB, Andreoni S, Martins P, Kozasa EH, Salvo VL, Sopezki D et al. Perceived stress among Primary Health Care Professionals in Brazil. Rev Bras Epidemiol. 2017;20(2):286-98. http://dx.doi.org/10.1590/19805497201700020009. PMid:28832851.

10. Moreira IJB, Horta JA, Duro LN, Chaves J, Jacques CS, Martinazzo $\mathrm{K}$ et al. Psychosocial aspects of work and psychological suffering among family health strategy workers. R Epidemiol Control Infec. 2017;7(1) http://dx.doi.org/10.17058/reci.v7i1.6927.

11. Ribeiro RP, Marziale MHP, Martins JT, Galdino MJQ, Ribeiro PHV Occupational stress among health workers of a university hospital Rev Gaúcha Enferm. 2018;39:e65127. http://dx.doi.org/10.1590/19831447.2018.65127. PMid:30043951.

12. Karasek RA, Theorell T. Healthy work: stress, productivity and the reconstruction of working life. New York: Basic Books; 1990

13. Hanson VF, Onasoga OA, Babalola C. Self-reported occupational stress, environment, working conditions on productivity and organizational impact among nursing staff in Nigerian hospitals. Int J Transl Med Res Public Health. 2017;1(2):29-35. http://dx.doi.org/10.21106/ijtmrph.50.

14. Ghareeb A, Said $\mathrm{H}$, El Zoghbi M. Examining the impact of accreditation on a primary healthcare organization in Qatar. BMC Med Educ. 2018;18(1):216. http://dx.doi.org/10.1186/s12909-018-1321-0. PMid:30236105.

15. Calvo MCM, Lacerda JT, Colussi CF, Schneider IJC, Rocha TAF Municipalities stratification for health performance evaluation. Epidemio Serv Saude. 2016 out;25(4):767-76. http://dx.doi.org/10.5123/S167949742016000400010. PMid:27869970.

16. Alves MGM, Chor D, Faerstein E, Lopes CS, Werneck GL. Short version of the "job stress scale": A Portuguese-language adaptation. Rev Saude Publica. 2004 abr;38(2):164-71. http://dx.doi.org/10.1590/ S0034-89102004000200003. PMid:15122370.

17. Fontana KC, Lacerda T, Machado MO. Work process in Primary Health Care: evaluation of management. Saúde Debate. 2016 set;40(110):6480. http://dx.doi.org/10.1590/0103-1104201611005.

18. Merhy EE, Feuerwerker LCM. Novo olhar sobre as tecnologias de saúde: uma necessidade contemporânea. In: Merhy EE, Baduy RS, Seixas ST, Almeida DES, Slomp Jr H, organizadores. Avaliação compartilhada do cuidado em saúde: surpreendendo o instituído nas redes. $1^{\underline{a}}$ ed. Rio de Janeiro: Hexis; 2016. p. 59-72.

19. Santos DS, Mishima SM, Merhy EE. Work process in Family Health Program: the potential of subjectivity of care for reconfiguration of the care model. Cien Saude Colet. 2018 mar;23(3):861-70. http://dx.doi. org/10.1590/1413-81232018233.03102016. PMid:29538566.

20. Macinko J, Harris MJ. Brazil's Family Health Strategy - delivering community-based primary care in a Universal Health System. N Engl Med. 2015;372(23):2177-81. http://dx.doi.org/10.1056/NEJMp1501140. PMid:26039598. 
21. Organização das Nações Unidas. Transformando nosso mundo: A Agenda 2030 para o Desenvolvimento Sustentável. Objetivo 3. Assegurar uma vida saudável e promover o bem-estar para todas e todos, em todas as idades [Internet]. Brasília, DF: ONU; 2015. [citado 2020 out 15]. Disponível em: https://nacoesunidas.org/pos2015/ods3/

22. Nicklin W, Fortune T, Van Ostenberg P, O'Connor E, McCauley N. Leveraging the full value and impact of accreditation. Int J Qual Health Care. 2017;29(2):310-2. http://dx.doi.org/10.1093/intqhc/mzx010. PMid:28453825.

23. Secretaria de Estado da Saúde do Paraná. Tutoria na Atenção Primária à Saúde. Manual operativo Selo Bronze [Internet]. Paraná; 2018. [citado 2020 out 15]. Disponível em: http://www.saude.pr.gov.br/arquivos/File/ ManualSeloBronze_2018_1.pdf

24. Higashi P, Simonetti JP, Carvalhaes MABL, Spiri WC, Parada CMGL. Potentially stressful situations for nurses considering the condition of accreditation of hospitals. Rev Rene. [Internet]. 2013 [citado 2020 out 15];14(6):1141-48. Disponível em: http://www.periodicos.ufc.br/rene/ article/view/3728

25. Salci MA, Paiano M, Radovanovic CAT, Carreira L, Meirelles BHS, Silva DMVG. Program of assessment of primary care from the perspective of health professionals and managers. Rev Rene. 2019;20:e33980. http:// dx.doi.org/10.15253/2175-6783.20192033980.

26. Silva JAM, Peduzzi M, Orchard C, Leonello VM. Interprofessional education and collaborative practice in Primary Health Care. Rev Esc Enferm USP. 2015 dez;49(spe2):16-24. http://dx.doi.org/10.1590/ S0080-623420150000800003. PMid:26959149.
27. Peduzzi M, Agreli HF. Teamwork and collaborative practice in Primary Health Care. Interface (Botucatu). 2018;22(2):1525-34. http://dx.doi. org/10.1590/1807-57622017.0827.

28. Oliveira JLC, Magalhães AMM, Bernardes A, Haddad MCFL, Wolff LDG, Marcon SS et al. Influence of hospital Accreditation on professional satisfaction of the nursing team: mixed method study. Rev Lat Am Enfermagem. 2019;27:e3109. http://dx.doi.org/10.1590/15188345.2799.3109.

29. Madsen IEH, Nyberg ST, Magnusson Hanson LL, Ferrie JE, Ahola K, Alfredsson $L$ et al. Job strain as a risk factor for clinical depression: systematic review and meta-analysis with additional individual participan data. Psychol Med. 2017;47(8):1342-56. http://dx.doi.org/10.1017/ S003329171600355X. PMid:28122650.

30. Silva MCN, Machado MH. Health and Work System: challenges for the Nursing in Brazil. Cien Saude Colet. 2020 jan;25(1):7-13. http://dx.doi. org/10.1590/1413-81232020251.27572019. PMid:31859850.

31. El Khamali R, Mouaci A, Valera S, Cano-Chervel M, Pinglis C, Sanz $C$ et al. Effects of a multimodal program including simulation on job strain among nurses working in intensive care units: a randomized clinical trial. JAMA. 2018;320(19):1988-97. http://dx.doi.org/10.1001/ jama.2018.14284. PMid:30357264.

32. Rocha GSA, Andrade MS, Silva DMRD, Terra MG, Medeiros SEG, Aquino JM. Feelings of pleasure of nurses working in primary care. Rev Bras Enferm. 2019;72(4):1036-43. http://dx.doi.org/10.1590/00347167-2018-0518. PMid:31432963. 